\title{
Ruthenium-Catalyzed Oxidative Cleavage of Alkynes to
}

\section{Carboxylic Acids}

\author{
Dan Yang, ${ }^{*} \dagger$, Fei Chen,${ }^{\dagger}$ Ze-Min Dong, ${ }^{\dagger}$ and Dan-Wei Zhang ${ }^{\S}$ \\ ${ }^{\dagger}$ Department of Chemistry, The University of Hong Kong, Pokfulam Road, Hong Kong, China, \\ ${ }^{\S}$ Department of Chemistry, Fudan University, Shanghai 200433, China \\ yangdan@hku.hk
}

\section{Supporting Information}

\section{Table of Contents:}

The preparation and characterization data of compounds $\mathbf{1 0}$ and $\mathbf{1 2}$

S2

${ }^{1} \mathrm{H}$ and ${ }^{13} \mathrm{C}$ NMR spectra of compounds $\mathbf{1 0}$ and $\mathbf{1 2}$

S3-S6 


\section{Preparation of 1-pent-3-ynyloxy-hexane (10)}

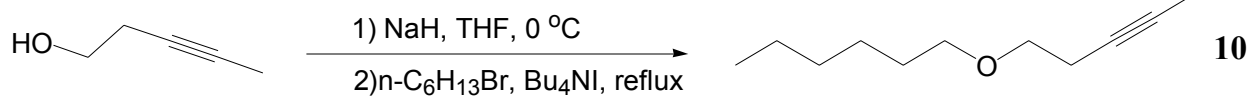

To a stirred suspension of $\mathrm{NaH}(60 \%, 0.78 \mathrm{~g}, 19.5 \mathrm{mmol})$ in dried $\mathrm{THF}(30 \mathrm{~mL})$ at $0{ }^{\circ} \mathrm{C}$ under nitrogen was added 3-pentyn-1-ol (1.52 g, $18 \mathrm{mmol})$. After $30 \mathrm{~min}, 1$-bromohexane (2.48 g, 15 $\mathrm{mmol})$ and tetrabutylammonium bromide $(0.167 \mathrm{~g}, 0.45 \mathrm{mmol})$ in THF $(15 \mathrm{~mL})$ were cannulated into the reaction mixture, and the reaction temperature was raised gradually to room temperature. After $1 \mathrm{~h}$, the temperature was heated to reflux for $6 \mathrm{~h}$. The reaction was quenched with saturated $\mathrm{NH}_{4} \mathrm{Cl}$ solution, and the solvent was removed in vacuo. The residue was diluted with $\mathrm{Et}_{2} \mathrm{O}$, washed with water and brine successively, dried over anhydrous $\mathrm{MgSO}_{4}$, concentrated in vacuo, and purified by flash chromatography to afford $10(1.55 \mathrm{~g}, 61 \%)$ as a colorless oil. ${ }^{1} \mathrm{H}$ NMR $\left(\mathrm{CDCl}_{3}, 300 \mathrm{MHz}\right): \delta 3.50(\mathrm{t}, J=7.2 \mathrm{~Hz}, 2 \mathrm{H}), 3.44(\mathrm{t}, J=6.7 \mathrm{~Hz}, 2 \mathrm{H}), 2.40(\mathrm{tq}, J=7.2,2.6 \mathrm{~Hz}$, 2H), $1.78(\mathrm{t}, J=2.5 \mathrm{~Hz}, 3 \mathrm{H}), 1.59-1.55(\mathrm{~m}, 2 \mathrm{H}), 1.34-1.25(\mathrm{~m}, 6 \mathrm{H}), 0.89(\mathrm{t}, J=6.3 \mathrm{~Hz}, 3 \mathrm{H})$; ${ }^{13} \mathrm{C} \mathrm{NMR}\left(75 \mathrm{MHz}, \mathrm{CDCl}_{3}\right) \delta 76.2,75.7,70.9,69.3,31.5,29.5,25.7,22.5,19.0,13.8,3.2$; IR $\left(\mathrm{CH}_{2} \mathrm{Cl}_{2}\right) 1108 \mathrm{~cm}^{-1}$; EI-MS (20 eV) $\mathrm{m} / z 153\left(\mathrm{M}^{+}-\mathrm{CH}_{3}, 37\right), 97$ (100), 85 (71); HRMS-EI $\mathrm{m} / z$ for $\mathrm{C}_{10} \mathrm{H}_{17} \mathrm{O}\left(\mathrm{M}^{+}-\mathrm{CH}_{3}\right)$ calcd 153.1279 , found 153.1276 .

\section{Preparation of tert-butyl-pent-3-ynyloxy-diphenyl-silane (12)}

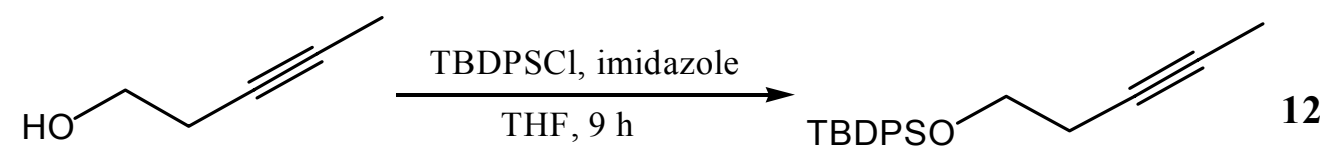

3-Pentyn-1-ol (0.252 g, $3 \mathrm{mmol})$, $t$-butylchlorodiphenylsilane (0.907 g, $3.3 \mathrm{mmol})$, dried THF $(10 \mathrm{~mL})$ and imidazole $(0.408 \mathrm{~g}, 6 \mathrm{mmol})$ were added sequentially into a $25 \mathrm{ml}$ flask. After stirring for $9 \mathrm{~h}$ at room temperature under nitrogen, the reaction was quenched with saturated $\mathrm{NH}_{4} \mathrm{Cl}$ solution, and diluted with $\mathrm{Et}_{2} \mathrm{O}$. The organic layer was washed with water and brine successively, dried over anhydrous $\mathrm{MgSO}_{4}$, concentrated in vacuo, and purified by flash chromatography to afford $12(0.96 \mathrm{~g}, 99 \%)$ as a colorless oil. ${ }^{1} \mathrm{H} \mathrm{NMR}\left(\mathrm{CDCl}_{3}, 400 \mathrm{MHz}\right)$ : $\delta 7.69-7.67(\mathrm{~m}, 4 \mathrm{H}), 7.44-7.35(\mathrm{~m}, 6 \mathrm{H}), 3.74(\mathrm{t}, J=7.2 \mathrm{~Hz}, 2 \mathrm{H}), 2.40$ (tq, $J=7.2,2.6 \mathrm{~Hz}, 2 \mathrm{H})$, $1.74(\mathrm{t}, J=2.6,3 \mathrm{H}), 1.05(\mathrm{~s}, 9 \mathrm{H}) ;{ }^{13} \mathrm{C} \mathrm{NMR}\left(100 \mathrm{MHz}, \mathrm{CDCl}_{3}\right) \delta 135.6,133.8,129.6,127.6$, 76.7, 76.1, 62.9, 26.8, 22.9, 19.1, 3.4; IR $\left(\mathrm{CH}_{2} \mathrm{Cl}_{2}\right)$ 3079, 1616, 1478, $1111 \mathrm{~cm}^{-1}$; EI-MS (20 eV) $m / z 266\left(\mathrm{M}^{+}-\mathrm{C}_{4} \mathrm{H}_{8}, 22\right), 265\left(\mathrm{M}^{+}-\mathrm{C}_{4} \mathrm{H}_{9}, 100\right)$; HRMS-EI $m / z$ for $\mathrm{C}_{17} \mathrm{H}_{17} \mathrm{OSi}\left(\mathrm{M}^{+}-\mathrm{C}_{4} \mathrm{H}_{9}\right)$ calcd 265.1049 , found 265.1048 . 

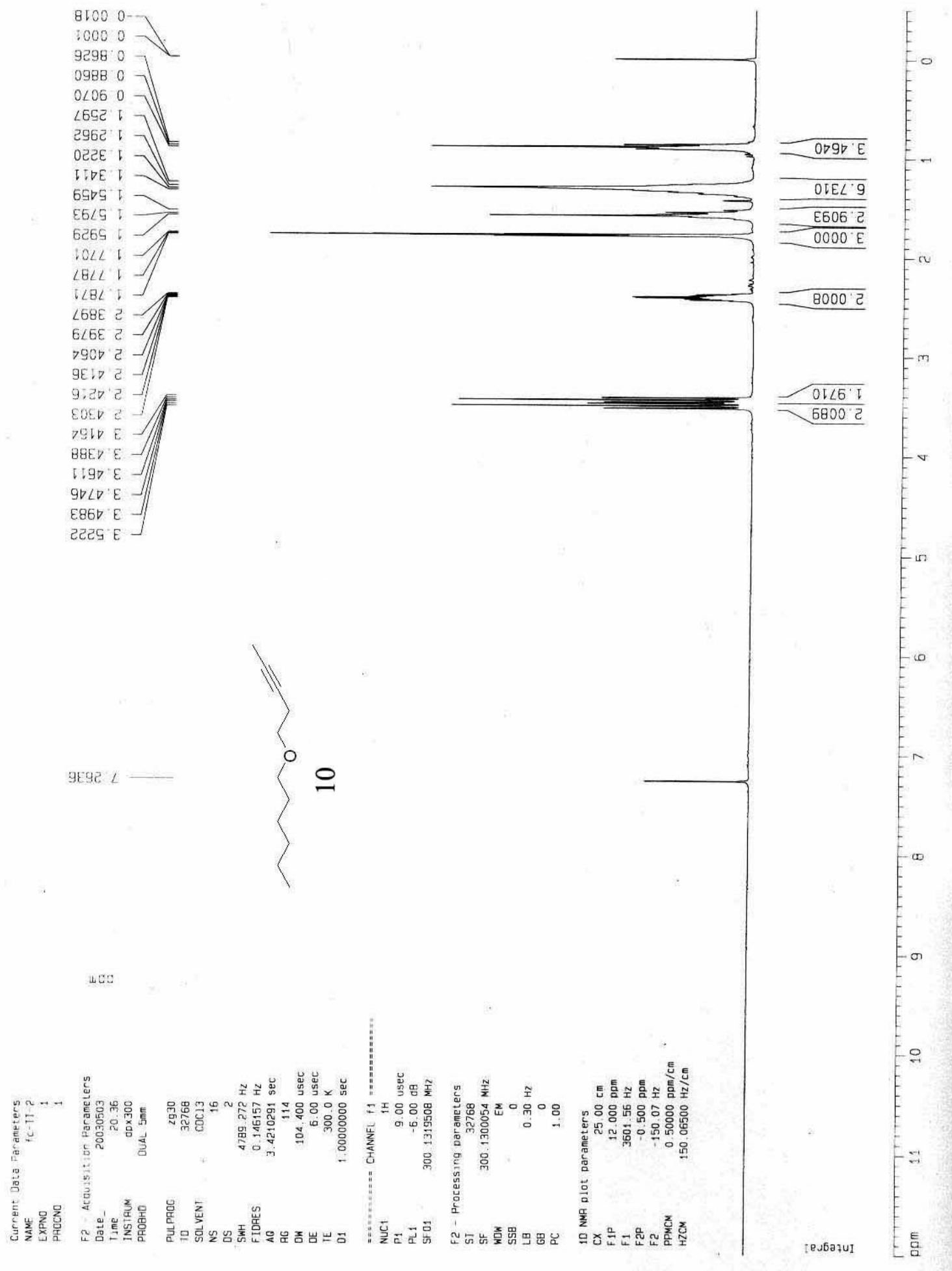
8ะธเ

686L EI-

$5106.61-$

$999725-$

$1509^{\circ} \mathrm{g} 2-$

$9199.92 \longrightarrow$

2989 $92-$

989762

20D5' IE

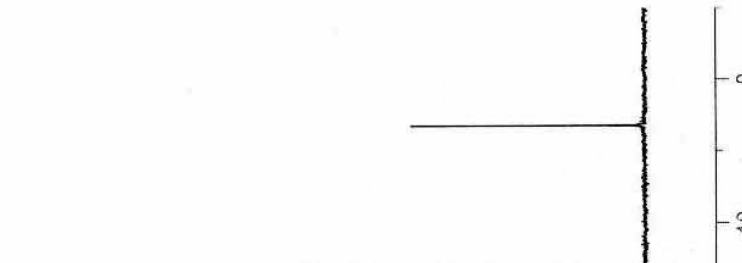

6I52 69

B878 02

$9<69 \cdot 5<-$

$90029 L-$

E189 $9 L-$

9666 $9 \angle-$

GLIE $\angle L-$
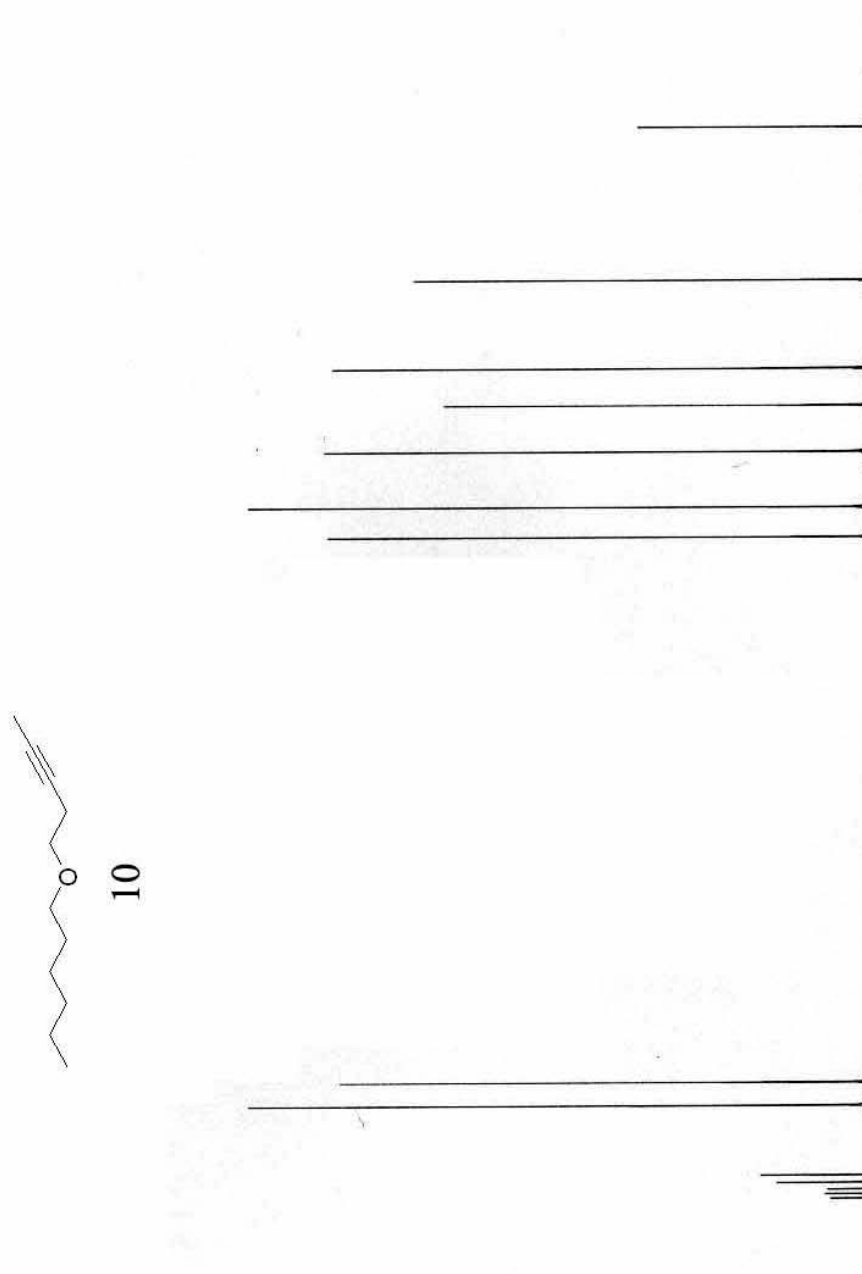

-ลำ

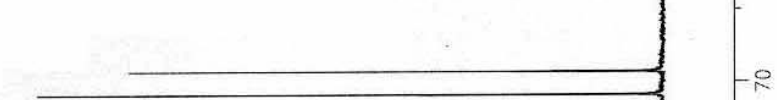

wdd

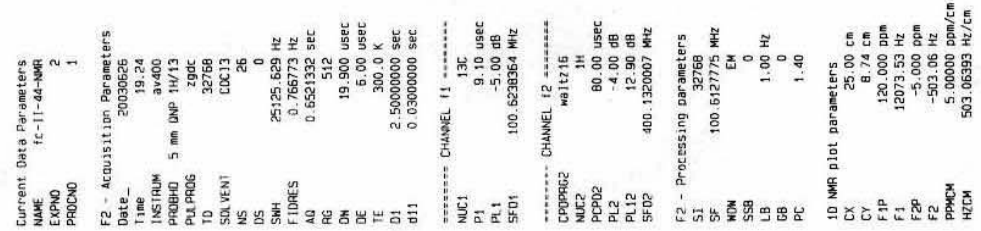




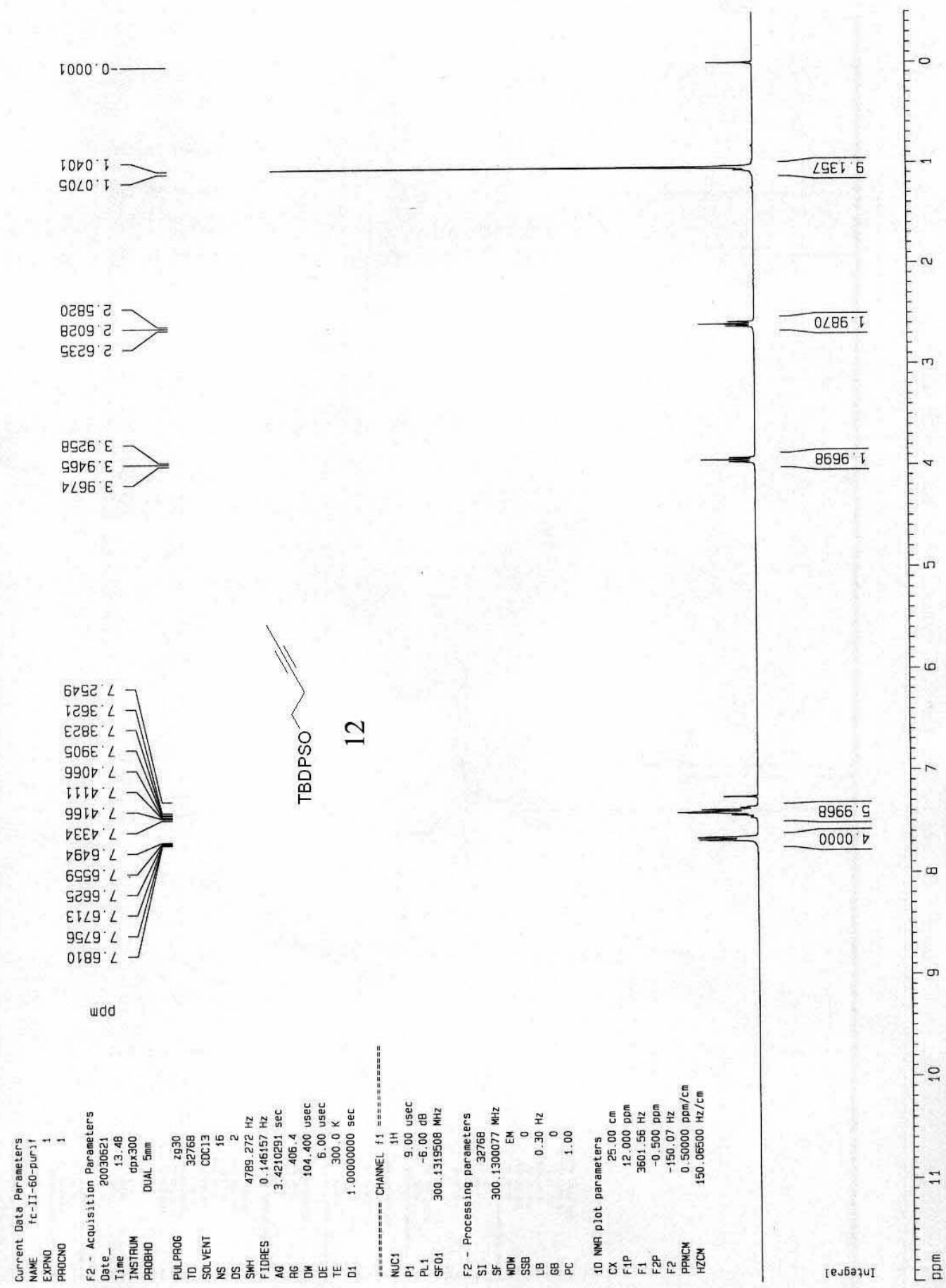




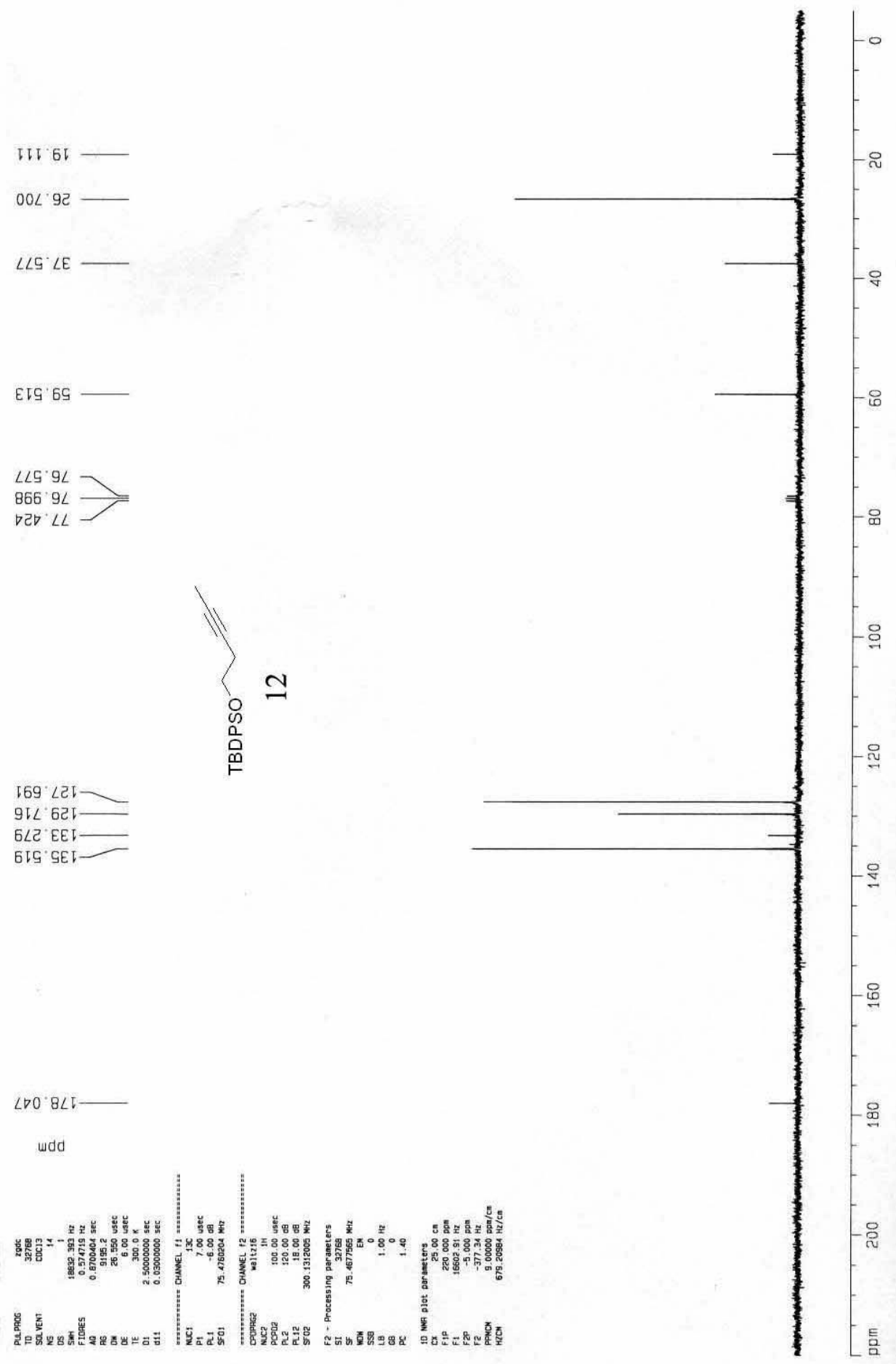

